\title{
Hyperfine anomaly in Be isotopes in the cluster model and the neutron spatial distribution.
}

\author{
Y. L. Parfenova and Ch. Leclercq-Willain \\ Physique Nucléaire Théorique et Physique Mathématique, CP229, \\ Université Libre de Bruxelles B 1050 Brussels, Belgium.
}

(Dated: June 28, 2018)

\begin{abstract}
The study of the hyperfine anomaly of neutron rich nuclei, in particular, neutron halo nuclei, can give a very specific and unique way to measure their neutron distribution and confirm a halo structure. The hyperfine structure anomaly in $\mathrm{Be}^{+}$ions is calculated with a realistic electronic wave function, obtained as a solution of the Dirac equation. In the calculations, the Coulomb potential modified by the charge distribution of the clustered nucleus and three electrons in the $1 s^{2} 2 s$ configuration is used. The nuclear wave function is obtained in the core+nucleon model of ${ }^{9,11} \mathrm{Be}$. The aim of this study is to test whether the hyperfine structure anomaly reflects a halo structure in ${ }^{11} \mathrm{Be}$.

PACS numbers: 32.10.Fn; 21.10.Gv; 21.60.Gx; 21.10.Ky
\end{abstract}

\section{INTRODUCTION}

Exotic (halo) nuclei are currently a subject of intensive experimental and theoretical studies. The interest in neutron-rich nuclei is to a large extent driven by experimental facilities and new experimental methods for studying the nuclear matter distribution. In particular, the ion trap method 11 and the NMR (Nuclear Magnetic Resonance) methods [2] allow the measurements of the hyperfine splitting of electronic states in atoms with an accuracy of the order $10^{-6}$, that provides the possibility of hyperfine anomaly studies.

The hyperfine splitting is sensitive to the magnetic current in the nucleus, and the hyperfine structure (hfs) constants extracted from the experimental data are related to the matter distribution of the nucleus. Therefore, the measurements of the hfs anomaly in neutron-rich halo nuclei can give a unique way to investigate the neutron distribution and the cluster structure.

For the experimental values of the magnetic moment of the Be isotopes, we refer to the measurements in Refs. $[3,4,5]$. But still there are no experimental data on the hyperfine anomaly for these nuclei.

A theoretical study has been performed by Fujita et al. [6] in order to verify how the halo structure manifests itself in the hyperfine structure. They calculated the hfs anomaly for Be isotopes both in the core+neutron model and in the single particle model, where the magnetic moment is well reproduced. They found that the hfs anomaly for ${ }^{11} \mathrm{Be}$ is large compared to that for the ${ }^{7,9} \mathrm{Be}$ isotopes, and that this was indicative of an extended neutron distribution and a halo structure in ${ }^{11} \mathrm{Be}$.

\footnotetext{
*Electronic address: Yulia.Parfenova@ulb.ac.be Also at Skobeltsyn Institute of Nuclear Physics, Moscow State University, 119992 Moscow, Russia

${ }^{\dagger}$ Electronic address: cwillain@ulb.ac.be
}

However, the approach used by Fujita et al. in Ref. [6] suffered from a poor knowledge of the ground state wave function of the ${ }^{9,11} \mathrm{Be}$ isotopes. The value of the hfs anomaly $\epsilon$ defined by the folding of the electronic and nuclear wave functions is rather sensitive to the spatial distribution of the valence neutron wave function and to the weights of the possible mixed states in the description of the ground state wave function. During the last decade new information on the ${ }^{11} \mathrm{Be}$ ground state wave function became available from the cross section measurements in the $p\left({ }^{11} \mathrm{Be},{ }^{10} \mathrm{Be}\right) d$ reaction and the weight of the $2 \mathrm{~s}_{1 / 2}$ state admixture was found at the value 0.84 [7] which is close to the theoretical estimation.

Besides this, the cluster (core+neutron) model while being rather good for the ${ }^{11} \mathrm{Be}$ nucleus, might fail for ${ }^{9} \mathrm{Be}$, which has essentially a three-cluster $(\alpha+\alpha+n)$ structure 8 . On the other hand, there is no strong evidence for the three-body structure of ${ }^{9} \mathrm{Be}$ in its ground state. So this is another interesting problem which can also be investigated in hfs anomaly studies.

The ${ }^{11} \mathrm{Be} 1 / 2^{+}$ground state can be represented by an admixture of the $2 \mathrm{~s}_{1 / 2}$ and $1 \mathrm{~d}_{5 / 2}$ valence neutron states, ${ }^{10} \mathrm{Be}\left(0^{+}\right) \otimes \nu\left(2 s_{1 / 2}\right)$ and ${ }^{10} \mathrm{Be}\left(2^{+}\right) \otimes \nu\left(1 d_{5 / 2}\right)$. The weight of the $s$ - state, related to the spectroscopic factor of this configurations obtained in both the shell model and the simple excited core cluster model, are found between 0.5 and 0.8 (for more information on the spectroscopic factors we refer to Refs. 9 , 10, 11]). In our calculations we take the weights of the states $w\left(2 s_{1 / 2} \otimes 0^{+}\right)=0.72$ and $w\left(1 d_{5 / 2} \otimes 2^{+}\right)=0.28$, consistent with the experimental data [12] (for more details, see [13]).

Let us mention that in Ref. [6] the hfs anomaly has been calculated within the Bohr-Weisskopf approach, where the electronic wave function is found for the interior of the nucleus. It is not applicable to the ${ }^{11} \mathrm{Be}$ nucleus, where a significant part (88\%) of the valence nucleon wave function is outside of the range of nuclear potential, indicating a halo [14]. In particular, the ${ }^{10} \mathrm{Be}$ core root mean square (rms) radius $2.61 \mathrm{fm}$ is rather 
small compared to the mean core-neutron distance (6 $\mathrm{fm})$. Thus, in Ref. [6] a rather simplified electronic wave function is used which significantly differs from the direct numerical solution of the Dirac equation in the region $r<10 \mathrm{fm}$. Besides this, in calculations of the electronic wave function, the electron screening effects of the Coulomb potential were not taken into account, although this effect can also be essential in the calculations of the hfs anomaly.

In the present paper, the relativistic electronic wave functions are calculated for the extended nuclear charge density distribution defined by the cluster structure of the nucleus [15]. We assume that two electrons in the $1 s$ state form the closed shell and are relatively unperturbed by the third electron in the $2 s$ state, and we define the wave function of this third electron as a solution of the Dirac equation for a nuclear charge potential screened by the $1 s^{2}$ electronic closed shell [16].

We compare our electronic wave function with that of Fujita et al. [6]. We analyze the hfs of ${ }^{9,11} \mathrm{Be}$ with regard to the available experimental information using more realistic descriptions of both the nuclear and the electronic wave functions. The aim of this paper is to answer the questions whether the hfs anomaly in these isotopes reflects the halo structure, and whether the hfs anomaly for ${ }^{11} \mathrm{Be}$ isotope is larger than for ${ }^{9} \mathrm{Be}$.

\section{MAGNETIC HYPERFINE STRUCTURE}

The magnetic hyperfine interaction Hamiltonian is defined by

$$
\mathcal{H}=-\int \mathbf{J}(\mathbf{r}) \cdot \mathbf{A}(\mathbf{r}) d^{3} r .
$$

Here, $\mathbf{J}$ is the nuclear current density, $\mathbf{A}$ is the vector potential created by the atomic electrons

$$
\mathbf{A}(\mathbf{r})=\frac{1}{c} \int \frac{\mathbf{j}\left(\mathbf{r}^{\prime}\right)}{\left|\mathbf{r}-\mathbf{r}^{\prime}\right|} d^{3} r^{\prime},
$$

where $\mathbf{j}\left(\mathbf{r}^{\prime}\right)$ denotes the electron current density operator

$$
\mathbf{j}\left(\mathbf{r}^{\prime}\right)=-e \alpha_{e} \delta\left(\mathbf{r}-\mathbf{r}^{\prime}\right)
$$

and $\alpha_{e}$ is the Dirac matrix for relativistic electrons.

For a $N$-electron system, the hyperfine interaction Hamiltonian can be defined as

$$
\mathcal{H}=-\frac{1}{c} \iint \frac{\mathbf{J}(r) \cdot \mathbf{j}\left(\mathbf{r}^{\prime}\right)}{\left|\mathbf{r}-\mathbf{r}^{\prime}\right|} d^{3} r d^{3} r^{\prime}
$$

With the Neumann expansion of $\frac{1}{\left|\mathbf{r}-\mathbf{r}^{\prime}\right|}$ in equation (4), the interaction Hamiltonian takes the form:

$$
\begin{aligned}
\mathcal{H} & =-\frac{1}{c} \iint \mathbf{J}(\mathbf{r}) \cdot \mathbf{j}\left(\mathbf{r}^{\prime}\right)\left[\mathbf{T}\left(\mathbf{r}_{<}\right) \cdot \mathbf{U}\left(\mathbf{r}_{>}\right)\right]_{\left(\mathbf{r} \mathbf{r}^{\prime}\right)} d^{3} r d^{3} r^{\prime} \\
& =+\frac{e}{c} \int \mathbf{J}(\mathbf{r}) \alpha_{e}\left[\mathbf{T}\left(\mathbf{r}_{<}\right) \cdot \mathbf{U}\left(\mathbf{r}_{>}\right)\right]_{\left(\mathbf{r r}_{e}\right)} d^{3} r
\end{aligned}
$$

where

$$
\begin{aligned}
\mathbf{T}\left(\mathbf{r}_{<}\right) \cdot \mathbf{U}\left(\mathbf{r}_{>}\right) & =\sum_{\lambda} \sum_{\nu} T_{\lambda \nu}\left(\mathbf{r}_{<}\right) U_{\lambda \nu}^{*}\left(\mathbf{r}_{>}\right) \\
& =\sum_{\lambda} \sum_{\nu}(-)^{\nu} \frac{r_{<}^{\lambda}}{r_{>}^{\lambda+1}} C_{\nu}^{\lambda}\left(\hat{r}_{<}\right) C_{-\nu}^{\lambda}\left(\hat{r}_{>}\right)
\end{aligned}
$$

and

$$
C_{\nu}^{\lambda}(\hat{r})=\sqrt{\frac{4 \pi}{2 \lambda+1}} Y_{\lambda \nu}(\hat{r})
$$

with $r_{<}$and $r_{>}$being the smallest and largest value of the nuclear $(r)$ or electronic $\left(r_{e}\right)$ coordinates. The $\lambda=1$ term is the magnetic dipole interaction between the magnetic field generated by the electrons and the nuclear magnetic dipole moment due to the extended nuclear matter distribution. The $\lambda=2$ term is the electric quadrupole interaction between the electric field gradient from the electrons and the non-spherical charge distribution of the nucleus.

The hyperfine interaction couples the electronic angular momentum $\mathbf{J}$ and the nuclear one $\mathbf{I}$ to an hyperfine momentum $\mathbf{F}=\mathbf{J}+\mathbf{I}$. The magnetic hyperfine splitting energy $W$ for a state $\mid I J F M_{F}=F>$ is defined as the matrix element of the Hamiltonian $\mathcal{H}$,

$$
\begin{aligned}
W_{(I J) F F} & =<I J F F|\mathcal{H}| I J F F> \\
& =\sum_{m, m^{\prime}}<I J F F|I M, J m><I M, J m| \mathcal{H}\left|I M^{\prime}, J m^{\prime}><I M^{\prime}, J m^{\prime}\right| I J F F>
\end{aligned}
$$

with the matrix element

$$
<I M, J m|\mathcal{H}| I M^{\prime}, J m^{\prime}>=-2 i \frac{e}{c} \sum_{\lambda}<I M\left|\int \mathbf{J}(\mathbf{r}) \cdot\left(\mathbf{A}_{\lambda}^{0}(r)+\mathbf{A}_{\lambda}^{c}(r)\right) d^{3} r\right| I M^{\prime}>
$$


where

$$
\mathbf{A}_{\lambda}^{0}(\mathbf{r})=r^{\lambda} \sum_{\nu}(-)^{\nu} C_{\nu}^{\lambda}(\hat{r}) \int_{0}^{\infty} d r_{e} \frac{r_{e}^{2}}{\left(r_{e}\right)^{\lambda+1}} F^{\kappa J}\left(r_{e}\right) G^{\kappa J}\left(r_{e}\right)<J m\left|C_{-\nu}^{\lambda}\left(\hat{r_{e}}\right) \sigma_{e}\right| J m^{\prime}>
$$

is the expression for a point nucleus, and

$$
\mathbf{A}_{\lambda}^{c}(\mathbf{r})=r \sum_{\nu}(-)^{\nu} C_{\nu}^{\lambda}(\hat{r}) \int_{0}^{r} d r_{e} F^{\kappa J}\left(r_{e}\right) G^{\kappa J}\left(r_{e}\right)\left(\frac{r_{e}^{\lambda+2}}{r^{\lambda+2}}-\frac{r^{\lambda-1}}{r_{e}^{\lambda-1}}\right)<J m\left|C_{-\nu}^{\lambda}\left(\hat{r}_{e}\right) \sigma_{e}\right| J m^{\prime}>
$$

is the correction term due to the finite extension of the nuclear density.

The functions $F^{\kappa J}, G^{\kappa J}$ are the radial parts of the large and small components of the Dirac wave function of the electron, with the quantum number $\kappa= \pm\left(J+\frac{1}{2}\right)$ for $J=L \mp \frac{1}{2}$ and the orbital angular momentum $L$.

In the dipole approach $(\lambda=1)$ the expression for $W_{(I J) F F}$ reduces to

$$
\begin{aligned}
W_{(I J) F F} & =<I J F F|\mathcal{H}| I J F F> \\
& =\frac{1}{2}[F(F+1)-I(I+1)-J(J+1)] a_{I}
\end{aligned}
$$

where $a_{I}$ is defined by

$$
a_{I}=-\frac{2 e \kappa \mu_{N}}{I J(J+1)}<I I\left|\sum_{i=1}^{A}\left(M_{Z}^{l}\left(\mathbf{r}_{i}\right)+M_{Z}^{s}\left(\mathbf{r}_{i}\right)\right)\right| I I>
$$

with the $Z$ components of the angular (or spin) magnetic moments $\mathbf{M}^{l(s)}\left(\mathbf{r}_{i}\right)$. Here, the summation runs over all nucleons.

Taking into account the density expansion of the nucleus, these moments are

$$
\begin{aligned}
& \mathbf{M}^{l}\left(\mathbf{r}_{i}\right)=g_{l}^{i} \mathbf{l}_{i}\left[\int_{r_{i}}^{\infty} F^{\kappa J} G^{\kappa J} d r+\int_{0}^{r_{i}} F^{\kappa J} G^{\kappa J}\left(\frac{r}{r_{i}}\right)^{3} d r\right], \\
& \mathbf{M}^{s}\left(\mathbf{r}_{i}\right)=g_{s}^{i}\left[\mathbf{s}_{i} \int_{r_{i}}^{\infty} F^{\kappa J} G^{\kappa J} d r+\mathbf{D}_{i} \int_{0}^{r_{i}} F^{\kappa J} G^{\kappa J}\left(\frac{r}{r_{i}}\right)^{3} d r\right],
\end{aligned}
$$

where $\mathbf{D}_{i}=-\sqrt{\frac{5}{2}}\left[\mathbf{s}^{1} \otimes C^{2}\left(\hat{r}_{i}\right)\right]^{1}$.

Thus $a_{I}$ can be expressed through the hfs constant for a point nucleus $a_{I}^{(0)}$ as

$$
a_{I} \cong a_{I}^{(0)}(1+\epsilon+\delta),
$$

where $\epsilon$ is defined as the hfs anomaly in the BohrWeisskopf effect and $\delta$ is the Breit-Rosenthal-CrawfordShawlow ('BRCS') correction [17].

The hfs constant for the point nucleus is

$$
a_{I}^{(0)}=-\frac{2 e \kappa \mu_{N} \mu}{I J(J+1)} \int_{0}^{\infty} F_{0}^{\kappa J}(r) G_{0}^{\kappa J}(r) d r
$$

where $\mu=<I I\left|\sum_{i=1}^{A} g_{s}^{i} s_{i}+g_{l}^{i} l_{i}\right| I I>$ defines the magnetic moment of the point nucleus in nuclear magneton units $\mu_{N}$. The functions $F_{0}^{\kappa J}(r), G_{0}^{\kappa J}(r)$ are the radial parts of the large and small components of the Dirac wave function for the electron in the point nucleus approximation.

So, the hfs anomaly is defined by

$$
\begin{aligned}
\epsilon & =-\frac{b}{\mu} \sum_{i=1}^{A}\left\{<I I\left|\left(g_{s}^{(i)} s_{i}+g_{l}^{(i)} l_{i}\right) K^{a}\left(r_{i}\right)\right| I I>\right. \\
& \left.-<I I\left|\left(g_{l}^{(i)} l_{i}+D_{i}\right) K^{b}\left(r_{i}\right)\right| I I>\right\} .
\end{aligned}
$$

Here, $b=\left[\int_{0}^{\infty} F_{0}^{\kappa J} G_{0}^{\kappa J} d r\right]^{-1}$ is a constant obtained from (10) and

$$
\begin{aligned}
K^{a}\left(r_{i}\right) & =\int_{0}^{r_{i}} F^{\kappa J} G^{\kappa J} d r \\
K^{b}\left(r_{i}\right) & =\int_{0}^{r_{i}} F^{\kappa J} G^{\kappa J}\left(\frac{r}{r_{i}}\right)^{3} d r .
\end{aligned}
$$

The 'BRCS' correction is $\delta=1-b K^{a}(\infty)$ [17].

Let us assume the two-cluster nuclear wave function defined as a superposition of different configurations $\Phi_{L, S}^{I}=\left[\Phi_{l, s_{c}}^{J_{c}} \otimes \varphi_{l, s}^{j}\right]^{I}$, associated to the coupling of a core state $\Phi_{l, s_{c}}^{J_{c}}$ and the valence particle wave function $\varphi_{l, s}^{j} . \quad J_{c}, s_{c}$ and $j, s$ are the total angular momentum and spin of the core fragment and valence nucleon, $l$ is the orbital angular momentum of their relative motion. For each configuration, the contribution to the hfs anomaly is given by

$$
\begin{aligned}
\epsilon & =-\frac{b}{\mu} \sum_{i=1,2}\left[<I I\left|\left(g_{s}^{(i)} s_{i}+g_{l}^{(i)} \frac{m_{3-i}}{M} l\right) K^{a}\left(\frac{m_{3-i}}{M} R\right)\right| I I>\right. \\
& \left.-<I I\left|\left(g_{l}^{(i)} \frac{m_{3-i}}{M} l+D_{i}\right) K^{b}\left(\frac{m_{3-i}}{M} R\right)\right| I I>\right]
\end{aligned}
$$

where indices correspond to the core $(i=1)$ and valence nucleon $(i=2), M=m_{1}+m_{2}$ is the mass of the whole system; $r$ and $R$ define, respectively, the electronic and relative radial coordinates of the two nuclear fragments. $g_{l}^{(i)}$ and $g_{s}^{(i)}$ are the gyromagnetic ratios of the $i$-th fragment orbital motion and spin, respectively. 


\section{ELECTRONIC WAVE FUNCTIONS}

In the experiment [1], the hyperfine splitting of the electronic levels $(F)$ is measured for the ground state of $\mathrm{Be}^{+}$ions. The electronic ground state of the $\mathrm{Be}^{+}$ion is represented by the $1 s^{2} 2 s$ configuration, and the hyperfine splitting of the states $F=2$ and $F=1$ is found at about $1.256 \mathrm{GHz}$.

In the present paper, we calculate the hfs anomaly of the Be isotopes using the electronic wave functions $F^{\kappa J}=\frac{f(r)}{r}$ and $G^{\kappa J}=\frac{g(r)}{r}$ obtained as solutions of the Dirac equation [18] taking into account the $\left(1 s^{2}\right)$ electron screening and the extended density of the nuclear charge distribution. The electronic wave functions in the region $r \leq 22 \mathrm{fm}$ are obtained for the Coulomb potential defined by the expression

$$
V(r)=4 \pi \frac{Z \alpha \hbar c}{r}\left\{\int_{0}^{r} \rho x^{2} d x+r \int_{r}^{\infty} \rho x d x\right\}
$$

where $\rho(x)$ is the charge distribution of the nucleus. This distribution is obtained in the cluster model of the Be isotopes using the ground state nuclear wave functions. To calculate the electronic wave functions we use the numerical methods suggested in Ref. [19].

The asymptotic electronic wave functions $(r \geq 22 \mathrm{fm})$ are found in the form

$$
\begin{aligned}
& f(r)=e^{-D r} \sum_{\nu}^{N} a_{\nu} r^{s+\nu} \\
& g(r)=\frac{s-k}{\alpha Z_{e f f}} e^{-D r} \sum_{\nu}^{N} b_{\nu} r^{s+\nu}
\end{aligned}
$$

where $A=\frac{1}{\hbar c}\left(E_{N}+m c^{2}\right), B=\frac{1}{\hbar c}\left(-E_{N}+m c^{2}\right), D=$ $\sqrt{A B}, s=\sqrt{k^{2}-\left(\alpha Z_{e f f}\right)^{2}}$ and the $2 s$ electronic energy $E_{N}$ given by

$$
E_{N}=m c^{2}\left[1+\left(\frac{Z_{e f f} \alpha}{N+\sqrt{k^{2}-\left(Z_{e f f} \alpha\right)^{2}}}\right)^{2}\right]^{-1 / 2}
$$

with $N=0,1,2 \ldots$, and $k= \pm 1, \pm 2, \pm 3 \ldots$, are found with the effective charge $Z_{\text {eff }}$ depending on radius $r . a_{\nu}$ and $b_{\nu}$ are expansion coefficients (see Ref. [18]).

In the case of $\mathrm{a} \mathrm{Be}^{+}$ion, there are three electrons orbiting the nucleus. We can make a simplification by considering the $2 s$ electron in the Coulomb potential of the nucleus screened by the closed electronic shell $1 s^{2}$. Thus, the asymptotic electron wave functions are defined for the screened Coulomb potential written in the form:

$$
\begin{aligned}
V(r) & =\frac{Z \alpha \hbar c}{r}\left[1-2 \pi\left\{\int_{0}^{r} \rho_{e l} x^{2} d x+r \int_{r}^{\infty} \rho_{e l} x d x\right\}\right. \\
& \left.+\frac{1}{2}(81 / 8 \pi)^{1 / 3} \rho_{e l}^{1 / 3}(r)\right]
\end{aligned}
$$

where $\rho_{e l}(x)$, is the electron density distribution of the two $1 s$ electrons in the closed shell. This potential can be approximated by

$$
V(r)=\frac{Z \alpha \hbar c}{r} \frac{1}{2}\left(e^{-\varkappa r}+1\right),
$$

where $\varkappa$ is fitted to reproduce the initial potential. So, the effective charge for the $2 s$ electron in the $\mathrm{Be}^{+}$ion is

$$
Z_{e f f}=\frac{Z}{2}\left(e^{-\varkappa r}+1\right)
$$

with $\varkappa=0.000068 \mathrm{fm}^{-1}$.

Finally, the electronic wave functions calculated in the interior of the nucleus are matched to those obtained with the screened Coulomb potential.

\section{RESULTS AND DISCUSSION}

The hfs anomaly $\epsilon$ for the $\mathrm{Be}^{+}$ion is obtained from the relation $a_{I}=a_{I}^{(0)}(1+\epsilon+\delta)$ where $a_{I}^{(0)}$ is the Fermicontact parameter found for a point nucleus [20]. In the core+nucleon model of the Be isotopes the values $a_{I}$ and $\epsilon$ are determined by the valence nucleon wave function (that, in general, is an admixture of different single particle states) and the electronic wave function depending on the charge distribution of the nucleus. Hence, the amount of clustering or the existence of a "halo" in the Be isotopes nuclei can be analyzed through the "hfs" anomaly evaluation.

\section{A. ${ }^{11} \mathbf{B e}$}

We consider the ${ }^{11} \mathrm{Be}$ nucleus as a two-body system composed of a ${ }^{10} \mathrm{Be}$ core nucleus in different states, $0^{+}$ and $2^{+}$, and a valence neutron. The ${ }^{11} \mathrm{Be}$ ground state is described by a superposition of the neutron states, $2 s_{1 / 2}$ and $1 d_{5 / 2}$, as

$$
\begin{aligned}
\left|{ }^{11} \operatorname{Be}\left(\frac{1}{2}^{+}\right)\right\rangle & =\beta\left|\left[{ }^{10} B e\left(0^{+}\right) \otimes n_{s_{1 / 2}}\right]_{1 / 2^{+}}\right\rangle \\
& +\lambda\left|\left[{ }^{10} B e\left(2^{+}\right) \otimes n_{d_{5 / 2}}\right]_{1 / 2^{+}}\right\rangle
\end{aligned}
$$

with the weights $w_{s}=\beta^{2}$ and $w_{d}=\lambda^{2}$ of the $s$ - and $d$ waves obtained in [12], which allow a good description of the ${ }^{11}$ Be interaction and break-up reaction cross sections at intermediate and high energies (see Ref. [13]).

In our approach, the magnetic moment of ${ }^{11} \mathrm{Be}$ in the ground state is

$$
\begin{aligned}
\mu & =w_{s} \mu_{s}+w_{d} \sum_{m_{s}, m_{l}} \sum_{m_{j}, M_{S}}\left(C_{s m_{s} l m_{l}}^{j m_{j}} C_{S M_{S} j m_{j}}^{\frac{1}{2} \frac{1}{2}}\right)^{2} \\
& \times\left[\frac{\mu_{d}}{1 / 2} m_{s}+\frac{\mu\left(2^{+}\right)}{2} M_{S}+\mu_{c} \frac{m_{l}}{2}\right] \\
& =w_{s} \mu_{s}+\frac{7}{15} w_{d} \mu_{d}+\frac{7}{15} w_{d} \mu_{c}-\frac{1}{3} w_{d} \mu\left(2^{+}\right)
\end{aligned}
$$


TABLE I: Core and valence neutron parameters used for the calculation of the core-nucleon wave function and core density parameters. $E_{x}$ is the excitation energy of the core state, $w_{j}$ are the weights of the different configurations , $V_{0}, R_{0}$ are the parameters of the Woods-Saxon potential with diffuseness parameter $a_{0}=0.5 \mathrm{fm}, B_{n}$ is the neutron separation energy.

\begin{tabular}{ccccccc}
\hline \hline$E_{x}$ & core & neutron & \multicolumn{3}{c}{ Woods-Saxon potential ${ }^{b}$} \\
\hline$(\mathrm{MeV})$ & $J_{c}^{\pi}$ & $n l_{j}$ & $w_{j}$ & $\begin{array}{c}V_{0} \\
(\mathrm{MeV})\end{array}$ & $\begin{array}{c}R_{0} \\
(\mathrm{fm})\end{array}$ & $\begin{array}{c}B_{n} \\
(\mathrm{MeV})\end{array}$ \\
\hline 0 & $0^{+}$ & $2 s_{1 / 2}$ & 0.719 & -70.83 & 2.483 & 0.504 \\
3.368 & $2^{+}$ & $1 d_{5 / 2}$ & 0.281 & -89.22 & 2.483 & 3.872 \\
\hline \hline
\end{tabular}

${ }^{b}$ The ${ }^{10}$ Be charge rms radius is related to the matter radius $r_{m}$ as $\mathrm{rms}=\sqrt{r_{m}^{2}+0.64}$, where $r_{m}$ is taken from Ref. [23]

where $\left(s, m_{s}\right),\left(l, m_{l}\right)$, and $\left(j, m_{j}\right)$ are the valence neutron spin, orbital angular momentum and total momentum, and their projections. $S, M_{S}$ are the spin of the ${ }^{10} \mathrm{Be}$ core nucleus and its projection. The magnetic moment of the ${ }^{10} \mathrm{Be}$ core fragment related to its orbital motion is $\mu_{c}=\frac{Z}{A(A-1)} l, A=11$. Here, $\mu_{s}, \mu_{d}$, and $\mu\left(2^{+}\right)$denote the magnetic moment of the neutron in the $s_{1 / 2}$ and $d_{5 / 2}$ states and the ${ }^{10} \mathrm{Be}$ core in the $2^{+}$state. We use the values $\mu_{s}=\mu_{d}=\frac{1}{2} g_{s}^{(n)}=-1.9135 \mu_{N}$. The magnetic moment of ${ }^{10} \mathrm{Be}$ in the excited state $I^{\pi}=2^{+}$has been calculated in Ref. 21] in the shell model, and has been found to be $\mu\left(2^{+}\right)=1.787 \mu_{N}$.

The ground state wave function (21) gives the ${ }^{11} \mathrm{Be}$ magnetic moment value $-1.784 \mu_{N}$ that is larger than the experimental value $\mu_{I}=-1.6816(8) \mu_{N}$ [5]. This problem with the magnetic moment description was discussed, in particular, in 22], and the weights of the $s$ and $d$ - waves have been found $w_{s}=0.55$, and $w_{d}=0.45$. In our simplified model, we reproduce the experimental value of the ${ }^{11} \mathrm{Be}$ magnetic moment with $w_{s}=0.5$, and $w_{d}=0.5$, which are the same as those used in calculations 6]. This example gives a measure of the sensitivity and the precision of the calculations.

The valence neutron wave function for each partial state in ${ }^{11} \mathrm{Be}$ is obtained as a solution of the Schrödinger equation with the same Woods-Saxon potential parameters as in Ref. 13], and reproduce the neutron separation energy $B_{n}$. These parameters are given in Table

The ${ }^{10} \mathrm{Be}$ core density is parametrized using the harmonic oscillator model [15]

$$
\rho(r)=\rho_{0}\left(1+\gamma(r / a)^{2}\right) \exp \left(-(r / a)^{2}\right),
$$

where $\rho_{0}$ is a normalization factor, $a=1.856 \mathrm{fm}$, and $\gamma=0.610$.

The charge density distribution of the ${ }^{11} \mathrm{Be}$ nucleus is defined as

$$
\rho(r)=\rho_{0} \int d^{3} \mathbf{x}\left|\Phi_{L}^{2}(x)\right| \rho_{c}\left(\left|\mathbf{r}-\frac{1}{11} \mathbf{x}\right|\right),
$$

where $\rho_{0}$ is a normalization factor, $\rho_{c}$ is the core density and $\Phi_{L}(x)$ defines the radial part of the wave function for the valence neutron. The charge density distribution in
(16) and the electronic density distribution in (18) give the Coulomb potential entering the Dirac equation for the wave function of the outer $2 s$ electron.

With the weights from Table $\square$ and parameters mentioned above we obtain the value of the hfs anomaly for the ${ }^{11} \mathrm{Be}^{+}$ions as

$$
\begin{aligned}
\epsilon & =-\frac{b}{\mu_{I}}\left[\beta^{2} \mu_{s}\left\{K_{0}^{a}\left(\frac{M_{c}}{M}\right)-\left(1-\Sigma_{\frac{1}{2}}\right) K_{0}^{b}\left(\frac{M_{c}}{M}\right)\right\}\right. \\
& \left.+\frac{7}{15} \lambda^{2} \mu_{d}\left\{K_{2}^{a}\left(\frac{M_{c}}{M}\right)-\left(1-\Sigma_{\frac{5}{2}}\right) K_{2}^{b}\left(\frac{M_{c}}{M}\right)\right\}\right] \\
& +\frac{1}{3} \frac{b}{\mu_{I}} \lambda^{2}\left\{\mu\left(2^{+}\right) \tilde{K}_{1}^{a}\left(\frac{m}{M}\right)+\Sigma\left(2^{+}\right) \tilde{K_{1}^{b}}\left(\frac{m}{M}\right)\right\} \\
& -\frac{7}{15} \lambda^{2} \mu_{c}\left\{K_{2}^{a}\left(\frac{M_{c}}{M}\right)-K_{2}^{b}\left(\frac{M_{c}}{M}\right)\right\},
\end{aligned}
$$

where

$$
\Sigma_{j}= \pm \frac{3}{4} \frac{j+1 / 2}{j+1}
$$

for $j=l \pm \frac{1}{2}$. The value of $\Sigma\left(2^{+}\right)$is taken as $\Sigma\left(2^{+}\right)=$ -1.034 (see Ref. [6]). Here, $m$ and $M_{c}$ are the mass of the neutron and of the core ${ }^{10} \mathrm{Be}$, respectively. The ratio $\frac{M_{c}}{M}$ $\left(\frac{m}{M}\right)$ takes into account the motion of the core (valence neutron) relative to the ${ }^{11} \mathrm{Be}$ center of mass $(\mathrm{cm})$.

The terms $K_{l}^{a}$ and $K_{l}^{b}$ are expressed as

$$
\begin{aligned}
K_{l}^{a}\left(m_{i}\right) & =\int_{0}^{\infty}\left|\Phi_{l}(R)\right|^{2} R^{2} d R K^{a}\left(m_{i} R\right) \\
K_{l}^{b}\left(m_{i}\right) & =\int_{0}^{\infty}\left|\Phi_{l}(R)\right|^{2} R^{2} d R K^{b}\left(m_{i} R\right)
\end{aligned}
$$

where $K^{a}$ and $K^{b}$ are defined in (13) and (14) and $\Phi_{l}(R)$ is the radial part of the valence nucleon wave function, for the state with the orbital angular momentum $l$.

Notice, that the terms $\tilde{K}_{1}^{a}$ and $\tilde{K}_{1}^{b}$ in (24) are calculated for the $p$ - wave nucleons composing the excited ${ }^{10} \mathrm{Be}$ nucleus and contributing to the magnetic moment of the excited $2^{+}$state. In these calculations we used the folding of the electronic part $K^{a(b)}$ with the ${ }^{10} \mathrm{Be}$ density distribution, assuming that the spatial distribution of the $p$ - wave nucleons is similar to the charge density distribution in ${ }^{10} \mathrm{Be}$.

Table III shows the values of the hfs anomaly calculated without screening effect $\left(\epsilon^{n s}\right)$, or that for a homogeneously charged sphere with the rms radius $2.61 \mathrm{fm}$ $\left(\epsilon^{h s}\right)$, and the value $\epsilon$ obtained by including all the effects. The contribution of the terms $\tilde{K}_{1}^{a}$ and $\tilde{K}_{1}^{b}$ in (24) to the hfs anomaly are given in the row named "core". In the Table, $r_{n}$ is the mean distance of the valence neutron from the ${ }^{11} \mathrm{Be} \mathrm{cm}$.

One can see from the Table, that the hfs anomaly is sensitive to the screening effect (about 25\%). The total value weakly depends on the shape of the charge density distribution (about $3 \%$ ), but the effect is different for 
TABLE II: The hfs anomaly in ${ }^{11} \mathrm{Be}^{+}$calculated for different states of the ${ }^{10} \mathrm{Be}$ core. $B_{n}$ is the neutron separation energy, $r_{n}$ is the neutron distance, $\epsilon^{n s}$ is the hfs anomaly value obtained without consideration of the screening, $\epsilon^{\text {hs }}$ is calculated for a homogeneous spherical charge distribution, and $\epsilon$ is obtained including all the effects. In the last two rows the weighted sums of the values are given.

\begin{tabular}{cccccccc}
\hline \hline $\begin{array}{c}\text { State } \\
n l_{j}\end{array}$ & $\begin{array}{c}B_{n} \\
(\mathrm{MeV})\end{array}$ & $\begin{array}{c}r_{n} \\
(\mathrm{fm})\end{array}$ & $\begin{array}{c}\epsilon^{n s} \\
(\%)\end{array}$ & $\begin{array}{c}\epsilon^{h s} \\
(\%)\end{array}$ & $\begin{array}{c}\epsilon \\
(\%)\end{array}$ & $\begin{array}{c}r_{n}{ }^{c} \\
(\mathrm{fm})\end{array}$ & $\begin{array}{c}\epsilon^{c} \\
(\%)\end{array}$ \\
\hline $2 s_{1 / 2}$ & 0.504 & 6.056 & -0.0558 & -0.0694 & -0.0719 & 6.165 & -0.120 \\
$1 d_{5 / 2}$ & 3.872 & 2.634 & -0.0090 & -0.0103 & -0.0112 & 3.551 & -0.0233 \\
core & 2.623 & -0.0006 & -0.0007 & -0.0008 & 2.588 & \\
\hline \multicolumn{2}{l}{ Total, $w_{s}=0.72$} & -0.0433 & -0.0534 & -0.0556 & & \\
\hline \multicolumn{2}{l}{ Total, $w_{s}=0.5$} & -0.0335 & -0.0410 & -0.0430 & & -0.0717 \\
\hline \hline
\end{tabular}

${ }^{c}$ cited from Ref. [6]

distinct partial states, and is more pronounced for the $d$ - wave neutron (about 8\%).

These values of $\epsilon$ can be compared with those from [6], $\epsilon\left(2 s_{1 / 2}\right)=-0.120 \%$ and $\epsilon\left(1 d_{5 / 2}\right)=-0.0233 \%$. The values from [6] are obtained without screening effect in a simplified model of the nuclear charge distribution, approximated by a homogeneously charged sphere with the radius $1.2 A^{\frac{1}{3}}=2.669 \mathrm{fm}$.

The value of the 'BRCS' correction is $\delta=0.0476 \%$ that is comparable with $\epsilon$.

The hfs anomaly value for each partial wave function strongly correlates with the neutron separation energies $B_{n}$ and $r_{n}$. Thus, the hfs anomaly value, obtained for the $s$-wave neutron is larger than that for the $d$-wave by an order of magnitude. Hence, the value of the hfs anomaly is very sensitive to the weights of the partial waves. For example, with the weights from Ref. [6] $w_{s}=w_{d}=$ 0.5 we get the value $\epsilon=-0.0430 \%$ which is essentially smaller than that obtained in Ref. [6], $\epsilon=-0.0717 \%$. The contribution of the core fragment is also small.

Thus, the precise measurement of the hfs anomaly might shed light upon the spectroscopic factors of the $s$ - and $d$ - states and the origin of the $\frac{1}{2}^{+}$state of ${ }^{11} \mathrm{Be}$.

The halo nucleus charge distribution might significantly deviate from a spherical distribution, defining the radial dependence of the electronic wave function. At the same time, the ${ }^{10} \mathrm{Be}-n$ mean distance is $6.7 \mathrm{fm}$, so that $r_{n} \simeq 6 \mathrm{fm}$, a value exceeding a few times the ${ }^{10} \mathrm{Be}$ core rms radius. Thus a significant part $(88 \%)$ of the valence nucleon wave function is outside of the range of the nuclear potential. Hence, the result of the folding of the electronic and nucleon wave functions essentially depends on the radial behavior of the wave functions.

Fig 1 shows the radial dependence of the electronic wave functions inside the ${ }^{11} \mathrm{Be}$ nucleus, calculated in the Bohr-Weisskopf approach 24], the simplified approach [6], and that obtained here as a numerical solution of the Dirac equation. One can see that the calculated wave functions are close to each other at $r \leq 3 \mathrm{fm}$ and significantly different at higher radii. For ${ }^{11} \mathrm{Be}$, the folding

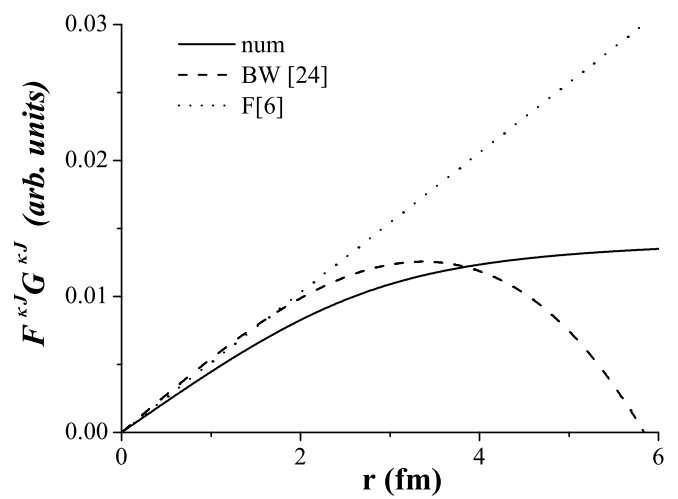

FIG. 1: Electronic wave functions $\left(J=\frac{1}{2}, \kappa=-1\right)$ inside the ${ }^{11}$ Be nucleus, obtained in the Bohr-Weisskopf approach [24] (BW - dashed line), the simplified approach of Fujita et al. 6] (F- dotted line), and the numerical solution of the Dirac equation (num - solid line).

of the electronic wave function with the wave function of the weakly bound valence nucleon gives significantly different results. Clearly, with the wave function of Fujita et al. [6], the hfs anomaly is overestimated for weakly bound nuclei.

This overestimation, in particular, can be seen from comparison of the hfs anomaly values for the $s$-wave valence neutron with close $r_{n}$ values. The result of our calculations is smaller than that from Ref. [6]. For the $d$-wave neutron, with different $r_{n}$ values we obtain the hfs anomaly value twice as small as that from Ref. [6]

Besides the difference in the electronic wave function radial dependence, the assessment 24] of the integrals with the electronic wave function in (25) and (26) as $K_{l}^{a}\left(\frac{m_{i}}{M}\right)-K_{l}^{b}\left(\frac{m_{i}}{M}\right)=C K_{l}^{a}\left(\frac{m_{i}}{M}\right)$ with $C=0.68$ is rather crude, and the coefficient $C$ for different partial waves varies within $20 \%$.

To compare the calculated value of the hfs anomaly in ${ }^{11} \mathrm{Be}$ with that for ${ }^{9} \mathrm{Be}$, as the next step of our analysis, we perform similar calculations for the ${ }^{9} \mathrm{Be}^{+}$ion.

\section{B. ${ }^{9} \mathrm{Be}$}

The hfs anomaly for ${ }^{9} \mathrm{Be}$ can be calculated within the two-cluster model of ${ }^{9} \mathrm{Be}$. This nucleus can be regarded as a system composed of a $p_{3 / 2}$-wave neutron and a ${ }^{8} \mathrm{Be}$ core in the ground $\left(0^{+}\right)$and excited $\left(2^{+}\right)$states.

Note, that in general, the states $0^{+}, 2^{+}$, and $4^{+}$are realized in ${ }^{8} \mathrm{Be}$, but only $0^{+}$and $2^{+}$contribute to the hfs anomaly in ${ }^{9} \mathrm{Be}$ (corresponding to the valence neutron angular momentum $l=1$ ). The contributions with $l=3$ are expected to be small.

The ${ }^{9} \mathrm{Be}$ ground state is given by a superposition of 
the $0^{+}$and $2^{+}$states

$$
\begin{aligned}
\left|{ }^{9} \mathrm{Be}\left(\frac{3^{-}}{2}\right)\right\rangle & =\beta\left|\left[{ }^{8} B e\left(0^{+}\right) \otimes n_{p_{3 / 2}}\right]_{3 / 2^{-}}\right\rangle \\
& +\lambda\left|\left[{ }^{8} B e\left(2^{+}\right) \otimes n_{p_{3 / 2}}\right]_{3 / 2^{-}}\right\rangle .
\end{aligned}
$$

With this wave function, denoting $w_{0^{+}}=\beta^{2}$ and $w_{2^{+}}=\lambda^{2}$, the magnetic moment of ${ }^{9} \mathrm{Be}$ in the core+cluster model is

$$
\mu=w_{0^{+}}\left\{\mu_{p}+\mu_{c}\right\}+w_{2^{+}}\left\{\frac{1}{5} \mu_{p}+\frac{3}{5} \mu_{c}+\frac{1}{5} \mu\left(2^{+}\right)\right\} .
$$

Here, indices in $w_{J_{c}^{\pi}}$ correspond to the core states $J_{c}^{\pi}=$ $0^{+}, 2^{+}$.

The magnetic moment of the ${ }^{8} \mathrm{Be}$ core related to its orbital motion is $\mu_{c}=\frac{Z}{A(A-1)} l, A=9$. Here, $\mu_{p}$, and $\mu\left(2^{+}\right)$denote the magnetic moment of the $p_{3 / 2}$ neutron and the ${ }^{8}$ Be core in the $2^{+}$state, $\mu_{p}=-1.9135 \mu_{N}$ and $\mu\left(2^{+}\right)=1$.

With the weights $w_{0^{+}}=0.535$ and $w_{2^{+}}=0.465$ obtained with the spectroscopic factors from Ref. [25] the magnetic moment is $\mu=-1.0687 \mu_{N}$. The change of the parameters to $w_{0^{+}}=0.579$ and $w_{2^{+}}=0.421$ allows one to reproduce the experimental value of the ${ }^{9} \mathrm{Be}$ magnetic moment $\mu_{I}=-1.1447 \mu_{N}$. This again gives a measure of the sensitivity of the calculated value to the weights of the states.

In our approach, these two states of ${ }^{9} \mathrm{Be}$ with the core in the ground and excited state are characterized by different neutron separation energy $B_{n}$ listed in Table III] For the case with ${ }^{9} \mathrm{Be}\left(0^{+}\right)$, the valence neutron wave function is obtained as a solution of the Schrödinger equation for the Woods-Saxon potential $V_{0}=-43.61 \mathrm{MeV}$, $a_{0}=0.5 \mathrm{fm}$, and $R_{0}=2.46 \mathrm{fm}$ giving $B_{n}=1.665 \mathrm{MeV}$ and the ${ }^{9}$ Be charge rms radius $2.519 \mathrm{fm}$.

In the case of excited ${ }^{8} \mathrm{Be}$ in the resonance state $2^{+}$, the parameters of the Woods-Saxon potential are taken as $V_{0}=-49.80 \mathrm{MeV}, a_{0}=0.5 \mathrm{fm}$, and $R_{0}=2.49 \mathrm{fm}$ to reproduce $B_{n}$ (see Table III).

The ${ }^{8}$ Be core density is parametrized in the harmonic oscillator model (23), with the parameters $a=1.749 \mathrm{fm}$ and $\gamma=0.619$ for the $0^{+}$state, and $a=1.768 \mathrm{fm}$ and $\gamma=0.624$ for the $2^{+}$state, respectively. The density parameters give the rms radius of the core equal to $R_{0}$ for each state.

The hfs anomaly for ${ }^{9} \mathrm{Be}^{+}$ion is

$$
\begin{aligned}
\epsilon & =\frac{b}{\mu_{I}}\left[\left(w_{0^{+}}+\frac{1}{5} w_{2^{+}}\right) \mu_{p}\left\{K_{1}^{a}\left(\frac{M_{c}}{M}\right)-\left(1-\Sigma_{\frac{3}{2}}\right) K_{1}^{b}\left(\frac{M_{c}}{M}\right)\right\}\right] \\
& +\frac{3}{5} \frac{b}{\mu_{I}} w_{2^{+}}\left\{\mu\left(2^{+}\right) \tilde{K}_{1}^{a}\left(\frac{m}{M}\right)+\Sigma\left(2^{+}\right) \tilde{K}_{1}^{b}\left(\frac{m}{M}\right)\right\} \\
& +\left(w_{0^{+}}+\frac{1}{5} w_{2^{+}}\right) \mu_{c}\left\{K_{1}^{a}\left(\frac{M_{c}}{M}\right)-K_{1}^{b}\left(\frac{M_{c}}{M}\right)\right\}
\end{aligned}
$$

The values $K_{l}^{a}$ and $K_{l}^{b}$ are calculated with (25) and (26). The values $\tilde{K}_{1}^{a}$ and $\tilde{K}_{1}^{b}$ are calculated with the density
TABLE III: The hfs anomaly $\epsilon$ in ${ }^{9} \mathrm{Be}^{+}$calculated for different states of the ${ }^{8} \mathrm{Be}$ core here and in [6], $w_{J_{c}^{\pi}}$ are the weights of the different configurations. $B_{n}$ is the neutron separation energy, $r_{n}$ is the neutron distance. In the last row the weighted sum of the values is presented.

\begin{tabular}{ccccccc}
\hline \hline $\begin{array}{c}\text { Core } \\
J_{c}^{\pi}\end{array}$ & $w_{J_{c}^{\pi}}$ & $\begin{array}{c}B_{n} \\
(\mathrm{MeV})\end{array}$ & $\begin{array}{c}r_{n} \\
(\mathrm{fm})\end{array}$ & $\begin{array}{c}\epsilon \\
(\%)\end{array}$ & $\begin{array}{c}r_{n}{ }^{a} \\
(\mathrm{fm})\end{array}$ & $\begin{array}{c}\epsilon^{a} \\
(\%)\end{array}$ \\
\hline $0^{+}$ & 0.535 & 1.665 & 3.200 & -0.0440 & 2.569 & -0.0249 \\
$2^{+}$ & 0.465 & 4.705 & 2.630 & -0.0066 & & \\
core & 0.535 & & 2.490 & 0.0063 & & -0.0249 \\
\hline Total & & \multicolumn{5}{c}{-0.0236} \\
\hline \hline
\end{tabular}

${ }^{a}$ cited from Ref. 6$]$

distribution of the core fragment in the ${ }^{9} \mathrm{Be}$ nucleus, taking into account the ${ }^{8}$ Be-neutron relative motion.

The calculations show that the contribution of the term $\Sigma\left(2^{+}\right) \tilde{K}_{1}^{b}\left(\frac{m}{M}\right)$ is small and we can neglect it.

In Table III the hfs anomaly values are listed for each ${ }^{8}$ Be state. The total value, obtained as the weighted sum is given in the last row of the Table. It is close to the values $\epsilon=-0.0249 \%$ [6], $\epsilon=-0.0243 \%$ [26].

The value of the 'BRCS' correction is $\delta=0.0451 \%$ that is larger than $\epsilon$.

One can see that the result for ${ }^{9} \mathrm{Be}$ is twice as small as that for ${ }^{11} \mathrm{Be}$. This corresponds to the conclusion in Ref. 6], that the value of the hfs anomaly reflects the extended neutron distribution in ${ }^{11}$ Be and might indicate a neutron halo. At the same time, the difference in the hfs anomaly values is not as large as in Ref. [6].

As with ${ }^{11}$ Be case, the ${ }^{9}$ Be hfs anomaly values for each partial wave function strongly correlate with the neutron distance $r_{n}$ from the Be $\mathrm{cm}$. In particular, the hfs anomaly obtained for the $p$-wave neutron for the $0^{+}$state of the ${ }^{8} \mathrm{Be}$ core is larger than that for the $2^{+}$state by an order of magnitude. The contribution of the core fragment is also small.

We should note, that we use a rather simplified model of ${ }^{9} \mathrm{Be}$ which most likely has a three-cluster structure $(\alpha+\alpha+n)$. In the three-cluster approach, there are partial states, contributing to the ${ }^{9}$ Be ground state wave function, which are not taken into account in the twocluster model, and which also contribute to the hfs anomaly value.

\section{CONCLUSION}

In the present paper, we have calculated the hfs anomaly in the ${ }^{9,11}$ Be isotopes. The nuclear wave functions have been calculated in the simplified core+neutron model. In calculations of the realistic electronic wave function, the charge distributions of the clustered nucleus and electrons in the $1 s^{2} 2 s$ configuration in $\mathrm{Be}^{+}$are taken into account.

It is found that the value of the hfs anomaly for ${ }^{11} \mathrm{Be}$ $\left(\epsilon=-0.0556 \%\right.$ for $w_{s}=0.72$ and $\epsilon=-0.0410 \%$ for 
$\left.w_{s}=0.5\right)$ is larger than that for ${ }^{9} \mathrm{Be}(\epsilon=-0.0235 \%)$, that agree with the conclusion of Fujita et al. [6].

For ${ }^{9}$ Be we reproduce the hfs anomaly value, obtained in [6], $\epsilon=-0.0249 \%$, and for ${ }^{11}$ Be we get the values with different weights $w_{s}$ of the $s$-wave, which are essentially less than that in [6], $\epsilon=-0.0717 \%$, obtained with the weight $w_{s}=0.5$.

In calculations of the hfs anomaly we did not consider the three-cluster structure of ${ }^{9} \mathrm{Be}$. We did not take into account the probable contribution of other states admixed in the ground state wave function of ${ }^{9} \mathrm{Be}$, which also might influence the calculated value of the anomaly. Thus, the difference in the hfs anomaly in the ${ }^{9} \mathrm{Be}$ and

${ }^{11} \mathrm{Be}$ isotopes might be even less.

Finally, we can conclude, that, on one hand, the hfs anomaly correlates with the neutron spatial distribution. As the anomaly value is sensitive to the ground state wave function of the nucleus, there is no unambiguous correlation between the hfs anomaly value and valence neutron distribution, and each nucleus requires a separate investigation.
[1] M. Wada, K. Okada, H. Wang, K. Enders, F. Kurth, T. Nakamura, S. Fujitaka, J. Tanaka, H. Kawakami, S. Ohtani, and I. Katayama, Nucl.Phys. A626, 365c (1997).

[2] ISOLDE Collaboration, H. T. Duong, C. Ekstrom, M. Gustafsson, T. T. Inamura, P. Juncar, P. Lievens, I. Lindgren, S. Matsuki, T. Murayama, R. Neugart, T. Nilsson, T. Nomura, M. Pellarin, S. Penselin, J. Persson, J. Pinard, I. Ragnarsson, O. Redi, H. H. Stroke, and J. L. Vialle, Nucl. Instr. and Methods in Phys. Research, Sect. A 325, 465 (1993).

[3] E. W. Weber and J. Vetter, Phys. Lett. 56A, 446 (1976).

[4] W. M. Itano, Phys. Rev. B 27, 1906 (1983).

[5] W. Geithner, S. Kappertz, M. Keim, P. Lievens, R. Neugart, L. Vermeeren, S. Wilbert, V. N. Fedoseyev, U. Koster, V. I. Mishin, V. Sebastian, and ISOLDE Collaboration, Phys. Rev. Lett. 83, 3792 (1999).

[6] T. Fujita, K. Ito, and T. Suzuki, Phys. Rev. C 59, 210 (1999).

[7] S. Fortier, S. Pita, J.S. Winfield, W. N. Catford, N. A. Orr, J. Van de Wiele, Y. Blumenfeld, R. Chapman, S. Chappell, N. M. Clarke, N. Curtis, M. Freer, S. Gales, K. L. Jones, H. Langevin-Joliot, H. Laurent, I. Lhenry, J. M. Maison, P. Roussel-Chomaz, M. Shawcross, M. Smith, K. Spohr, T. Suomijarvi, and A. de Vismes, Phys. Lett. 461B, 22 (1999).

[8] P. Descouvemont, Nucl. Phys. A699, 463 (2002).

[9] E. K. Warburton and B.A. Brown, Phys. Rev. C 46, 923 (1992).

[10] F. M. Nunes, J. A. Christley, I. J. Thompson, R. C. Johnson, and V. D. Efros, Nucl. Phys. A609, 43 (1996).

[11] D. L. Auton, R. G. H. Robertson, Nucl. Phys.A157, 305 (1970).
[12] T. Aumann, A. Navin, D. P. Balamuth, D. Bazin, B. Blank, B. A. Brown, J. E. Bush, J. A. Caggiano, B. Davids, T. Glasmacher, V. Guimaraes, P. G. Hansen, R. W. Ibbotson, D. Karnes, J. J. Kolata, V. Maddalena, B. Pritychenko, H. Scheit, B. M. Sherrill, and J. A. Tostevin, Phys. Rev. Lett. 84, 35 (2000).

[13] Yu. L. Parfenova, M. V. Zhukov, and J. S. Vaagen, Phys. Rev. C 62, 044602 (2000).

[14] D.V. Fedorov, A.S. Jensen, and K. Riisager, Phys. Lett. 312B, 1 (1993).

[15] H. de Vries, C. W. de Jager, and C. de Vries, At. Data and Nucl. Data Tables 36, 495 (1987).

[16] F. Rösel, H. M. Fries, K. Adler, and H. C. Pauli, At. Data and Nucl. Data Tables 21, 92 (1978).

[17] H.J. Rosenberg and H.H. Stroke, Phys. Rev. A 5, 1992 (1972).

[18] A.S. Davydov, "Quantum Mechanics", Pergamon Press, Oxford, London, Edinburg, New York, Paris, Frankfurt, 1965.

[19] M.E. Rose "Relativistic Electron Theory", John Wiley and Sons, New York, 1961.

[20] M. Le Bellac, Nucl. Phys. 40, 645 (1963).

[21] T. Suzuki, T. Otsuka, and A. Muta, Phys. Lett. 364B, 69 (1995).

[22] T. Otsuka, N.Fukunishi, and H. Sagawa. Phys. Rev. Lett. 70, 1385 (1993).

[23] A.Ozawa, T. Suzuki, and I. Tanihata, Nucl. Phys. A693, 32 (2001).

[24] A. Bohr and V.F. Weisskopf, Phys. Rev. 77, 94 (1950).

[25] S. Cohen and D. Kurath, Nucl. Phys. A101, 1 (1967).

[26] N. Yamanaka, Hyperfine interactions 127, 129 (2000). 